\title{
Should family members witness cardiopulmonary resuscitation?
}

\author{
Reviewed by Salvatore Mottillo, MD*; J. Scott Delaney, $\mathrm{MDCM}^{\dagger}$
}

\begin{abstract}
Clinical question
What is the effect of family presence during cardiopulmonary resuscitation on family members and the medical team?

Article chosen

Jabre P, Belpomme V, Azoulay E, et al. Family presence during cardiopulmonary resuscitation. $N$ Engl $J$ Med 2013;368:1008-18.

\section{Objective}

The authors sought to determine whether systematically offering relatives the option to be present during cardiopulmonary resuscitation increases the proportion of relatives with posttraumatic stress disorder-related symptoms after 90 days. Secondary outcomes included the presence of anxiety and depression symptoms in relatives, the effect of family presence on medical efforts at resuscitation, the well-being of the medical team, and the occurrence of medicolegal claims.
\end{abstract}

Keywords: cardiopulmonary resuscitation, family presence, posttraumatic stress disorder

\section{BACKGROUND}

Witnessing cardiopulmonary resuscitation $(\mathrm{CPR})$ on a family member can be an incredibly stressful experience. Family members who are present during CPR are at high risk for posttraumatic stress disorder (PTSD), anxiety, and depression. ${ }^{1}$ Surveys have shown that physicians are often reluctant to have relatives witness CPR. ${ }^{2,3}$ In addition to the possible psychological harm to family members, it has been argued that family presence may interfere with medical efforts and may result in medicolegal claims. ${ }^{4-6}$

Proponents for family presence during CPR argue that witnessing CPR may allow relatives to understand the resuscitation process and the chance to bid farewell to their loved one. ${ }^{7,8}$ The psychological benefits in relatives who have witnessed $\mathrm{CPR}$ have only been demonstrated in small surveys, observational studies, ${ }^{4,8-13}$ and a small randomized controlled trial (RCT). ${ }^{14}$ Despite the limited evidence, several major international guidelines support family presence during CPR. ${ }^{15-17}$

In the study reviewed, Jabre and colleagues sought to determine whether systematically offering relatives the option to be present during out-of-hospital CPR increases the proportion of PTSD-related symptoms, anxiety, and depression. ${ }^{18}$ They also aimed to clarify the effect of family presence on the medical team and resuscitation effort.

\section{STUDY DESIGN}

This was a prospective, cluster-RCT conducted in 15 prehospital emergency medical service (EMS) units in France. Eight and seven participating units were randomized to the intervention and control groups, respectively. Adult family members of adult patients in cardiac arrest were enrolled. Only one first-degree relative per patient participated. Relatives were excluded if there were communication barriers or if CPR was not attempted. Family members in the intervention group were systematically asked by a

From the *McGill University Emergency Medicine Residency Program, Royal Victoria Hospital, and the tDepartment of Emergency Medicine, McGill University Health Centre, Montreal, QC.

Correspondence to: Dr. Salvatore Mottillo, McGill University Emergency Medicine Residency Program, Royal Victoria Hospital, Suite A4.62, 687 Pine Avenue West, Montreal, QC H3A 1A1; salvatore.mottillo@mail.mcgill.ca.

This article has been peer reviewed. 
member of the medical team whether they wished to witness the CPR and, if so, would be taken to the room, usually in the home, where the CPR was performed. Furthermore, an assigned physician used a communication guide to coach relatives throughout the resuscitation procedure and for the pronouncement of death. In contrast, family members in the control group were not routinely given the option to witness the CPR. Physician team leaders interacted with these relatives in a standard manner during CPR.

\section{OUTCOME MEASURES}

The primary outcome was the proportion of relatives experiencing PTSD-related symptoms at 90 days (score $>30$ on the Impact of Event Scale [IES]). ${ }^{19} \mathrm{~A}$ trained psychologist, blinded to the study group assignments, conducted the IES by telephone. Secondary outcomes included the presence of anxiety or depression (score $>10$ on the Hospital Anxiety and Depression Scale [HADS]), ${ }^{20}$ the effect of family presence on medical efforts, the well-being of the medical team, and medicolegal claims. An intentionto-treat analysis was used for the primary outcome. Relatives who did not complete the IES questionnaire because of emotional distress were classified as having PTSD-related symptoms. Multiple imputation was used for missing data.

\section{RESULTS}

A total of 570 family members were enrolled, of whom 266 were randomized to the intervention (systematically asked if they wanted to be present) and 304 to the control (usual care). At 90 days, 95 family members (17\%) did not complete the IES questionnaire. The baseline characteristics for patients and relatives, as well as characteristics of the resuscitation procedure and survival, did not differ significantly between the study groups.

The frequency of PTSD-related symptoms was significantly higher in the control group than in the intervention group (adjusted odds ratio 1.7; 95\% confidence interval [CI] 1.2-2.5) and was significantly higher among family members who did not witness CPR than among those who did (adjusted odds ratio 1.6; 95\% CI 1.1-2.5). Symptoms of anxiety were also more frequent in the control than in the intervention group ( $n=55$ of 239 [23\%] v. $n=34$ of 230
$[15 \%] ; p<0.001)$. Twelve percent of relatives who did not witness CPR expressed regret at having been absent versus only $3 \%$ of relatives who did witness CPR who regretted being present. Less than $1 \%(n=4$ of 507) of relatives were aggressive or in conflict with the medical team, and there was no significant difference in stress levels in the medical team according to family presence. There were no medicolegal claims in either group.

\section{STUDY CONCLUSION}

The authors concluded that systematically offering family members the option to be present during CPR was associated with positive results on psychological variables and did not hinder medical efforts, increase stress in the medical team, or result in medicolegal claims.

\section{COMMENTARY}

As recently as 40 years ago, fathers were not welcome to accompany their spouse during childbirth. In the medical field, it was common belief that fathers would be traumatized by the sight of childbirth or would interfere with medical efforts. ${ }^{21-23}$ Now such notions seem preposterous. Are we making similar erroneous assumptions with the presence of family during CPR?

Jabre and colleagues sought to clarify whether relatives should be asked to be present during CPR. This study showed that the frequency of PTSD-related symptoms was significantly higher in the control group than in the intervention group and among family members who did not witness CPR than among those who did. We identified one small $\mathrm{RCT}^{14}$ and two prospective cohort studies ${ }^{4,11}$ that had previously examined psychological variables in relatives postCPR. In the previous RCT $(N=25)$, relatives who did not witness CPR obtained a median IES score of 26.5 (interquartile range [IQR] 17.3-35.1) versus 23.5 (IQR 14.8-42.8) for relatives witnessing CPR. ${ }^{14}$ The authors were convinced of the better scores in the relatives witnessing CPR and ended the trial early. Their results never reached significance due to the small sample size.

Of the two prospective cohort studies identified, the authors of one study demonstrated no harmful effects on anxiety, satisfaction, and well-being in relatives who were present at the bedside during trauma resuscitation $(N=50) .{ }^{11}$ However, the authors of another 
prospective study $(N=54)$ suggested that PTSDrelated symptoms may be higher in relatives witnessing CPR when calculating the mean difference in PTSD symptom scores $(6.87 ; 95 \%$ CI $0.57-13.17) .{ }^{4}$ Several other small descriptive surveys have been previously performed and concluded that relatives who witnessed CPR experienced fewer symptoms of anxiety and depression..$^{8-10,12,13}$

Several major international guidelines, including those of the American Heart Association (AHA), recommend inviting family members to witness CPR. ${ }^{15-17}$ As few as $8 \%$ of Canadian hospitals have guidelines for the presence of family during resuscitation. ${ }^{24}$ Much of the literature on family-witnessed CPR has focused on the attitudes and beliefs of family members and health care workers. ${ }^{7,25-28}$ In these surveys, the majority of family members preferred to be present during CPR. Similarly, in the study by Jabre and colleagues, of patients given the option to witness CPR, $79 \%$ chose to be present. However, a review of health care worker surveys demonstrated that a large proportion of physicians are reluctant to have family presence. ${ }^{28}$ The most notable reasons for this reluctance are the beliefs that witnessing CPR would negatively impact the psychological variables in relatives, lead to family member interference with the medical effort, and increase medicolegal issues.

Jabre and colleagues attempted to address these concerns in their secondary analyses. Of patients who were present during CPR, few were aggressive or in conflict with the medical team. The median stress score of emergency physicians did not differ according to family presence. When comparing family presence versus family absence during CPR, there were no differences in the median defibrillation attempts $(3$; [IQR 1-5] v. 4; [IQR 1-6]; $p=0.56$ ), epinephrine administration (7; [IQR 5-10] v. 7; [IQR 5-10]; $p=$ 0.86 ), and duration of resuscitation (30; [IQR 23-40] v. 30; [IQR 20-40]; $p=0.58$ ).

This study provided strong evidence supporting family-witnessed CPR. Although it challenged the belief elicited from physician surveys that physicians tend to be uncomfortable with family-witnessed $\mathrm{CPR},{ }^{2,3}$ its results were in concordance with most previous small prospective and descriptive studies examining PTSD, depression, and resuscitation outcomes. $^{8-12,14}$ There were several strengths to this study, including its randomized controlled design and large sample size $(N=570)$. There were few exclusion criteria limiting selection bias, and baseline characteristics were similar between the two groups. Outcome assessment was appropriately implemented by a blinded psychologist, and an intention-to-treat analysis was conducted.

There are several elements to consider when interpreting the results of this study. First, subjects were randomized according to the choice to witness CPR. The intervention consisted of systematically asking relatives if they wanted to be present, and the control consisted of usual care. As a result, not all subjects were present during CPR in the intervention group or absent during CPR in the control group. There was significant overlap between the groups, with $79 \%$ and $43 \%$ of relatives witnessing CPR in the intervention and control groups, respectively. Secondary analyses comparing relatives who witnessed CPR to relatives who did not witness CPR should be interpreted with caution as subjects were not randomized in this way.

Second, there were a number of elements that may have limited the internal validity of this study. There may have been differences in the care provided to the intervention and control groups. Relatives in the intervention group were accompanied by an investigator who explained the resuscitative process. The lead physician met with the family and used a communication guide to help with the pronouncement of death. The differences in PTSD-related symptoms may not have been solely due to witnessing CPR but rather how the relatives were treated by the physician involved. There was also a risk of attrition bias because $17 \%$ of relatives $(20.4 \%$ v. $12.4 \%$ in the control and intervention groups, respectively) did not complete the outcome assessment at 90 days. The authors employed an intention-to-treat analysis and attempted to limit this bias by using multiple imputation.

Third, the external validity of this study is limited. The study was conducted for out-of-hospital cardiac arrests, usually in the home of the patient. The generalizability of the results to in-hospital arrests may be limited as witnessing CPR in the hospital may be more traumatic for family members, or, conversely, the home may be a constant reminder of a traumatic event. Furthermore, the EMS system in France differs from the North American system. French ambulances actually resemble mobile intensive care units and are staffed with physicians. From a medicolegal standpoint, medicolegal claims are less frequent in France than in North America. ${ }^{29}$ 
Fourth, this study may have benefitted from subgroup analyses to determine the psychological impact of family-witnessed CPR in different circumstances for cardiac arrest, including trauma, suicide, and expected death. PTSD-related symptoms may be higher in relatives present during a trauma, for which CPR conditions may be less than ideal. Stress levels for health care workers with varying levels of experience may also be different. A subset of patients experienced return of spontaneous circulation $(30.0 \%)$ and survival at 28 days (3.9\%). Subgroup analyses may have helped determine whether PTSD-related symptoms differed for the relatives of these patients.

Finally, the follow-up time for the primary outcome of this study was 90 days. The development of PTSDrelated symptoms, anxiety, or depression following a loss may occur several months later. Although the process of bereavement varies between individuals, the stages of bereavement from death of a loved one usually last at least 6 months. ${ }^{30}$ PTSD-related symptoms may be delayed up to 6 months after a traumatic event. ${ }^{31}$ The follow-up period may have been too short to detect all cases of PTSD-related symptoms after CPR.

\section{CONCLUSION}

This RCT provides valuable information supporting the AHA recommendation to offer family members the option to be present during CPR. Systematically offering family members the option to be present during CPR was associated with fewer PTSD-related symptoms and was not harmful for any of the stakeholders. Previous surveys of physicians have demonstrated a reluctance to provide family members with the option of being present during CPR. Future studies with longer follow-up periods, performed in other countries, and conducted within the hospital may be needed to minimize this reluctance. Perhaps in the future, family members will be welcomed to the resuscitation bay as fathers are now welcomed in the delivery room.

Competing interests: None declared.

\section{REFERENCES}

1. Christakis NA, Allison PD. Mortality after the hospitalization of a spouse. $N$ Engl 7 Med 2006;354:719-30, doi:10. 1056/NEJMsa050196.
2. Helmer SD, Smith RS, Dort JM, et al. Family presence during trauma resuscitation: a survey of AAST and ENA members. American Association for the Surgery of Trauma. Emergency Nurses Association. 7 Trauma 2000;48:101522; discussion 1023-4, doi:10.1097/00005373-20000600000004

3. McClenathan BM, Torrington KG, Uyehara CF. Family member presence during cardiopulmonary resuscitation: a survey of US and international critical care professionals. Chest 2002;122:2204-11, doi:10.1378/chest.122.6.2204.

4. Compton S, Grace H, Madgy A, Swor RA. Post-traumatic stress disorder symptomology associated with witnessing unsuccessful out-of-hospital cardiopulmonary resuscitation. Acad Emerg Med 2009;16:226-9, doi:10.1111/j.1553-2712. 2008.00336.x.

5. Mian $\mathrm{P}$, Warchal S, Whitney $\mathrm{S}$, et al. Impact of a multifaceted intervention on nurses' and physicians' attitudes and behaviors toward family presence during resuscitation. Crit Care Nurse 2007;27:52-61.

6. Tsai E. Should family members be present during cardiopulmonary resuscitation? N Engl f Med 2002;346:1019-21, doi:10.1056/NEJM200203283461312.

7. Howlett MS, Alexander GA, Tsuchiya B. Health care providers' attitudes regarding family presence during resuscitation of adults: an integrated review of the literature. Clin Nurse Spec 2010;24:161-74, doi:10.1097/NUR.0b013e3181 dc548a.

8. Doyle CJ, Post H, Burney RE, et al. Family participation during resuscitation: an option. Ann Emerg Med 1987;16: 673-5, doi:10.1016/S0196-0644(87)80069-0.

9. Belanger MA, Reed S. A rural community hospital's experience with family-witnessed resuscitation. 7 Emerg Nurs 1997;23:238-9, doi:10.1016/S0099-1767(97)90015-5.

10. Meyers TA, Eichhorn DJ, Guzzetta CE, et al. Family presence during invasive procedures and resuscitation. $A m \mathcal{F}$ Nurs 2000;100:32-42; quiz 43.

11. Pasquale MA, Pasquale MD, Baga L, et al. Family presence during trauma resuscitation: ready for primetime? $\mathcal{7}$ Trauma 2010;69:1092-9; discussion 1099-100, doi:10.1097/TA.0b0 $13 \mathrm{e} 3181 \mathrm{e} 84222$.

12. Holzhauser K, Finucane J, De Vries S. Family presence during resuscitation: a randomised controlled trial of the impact of family presence. Australas Emerg Nurs 7 2006;8: 139-47, doi:10.1016/j.aenj.2005.10.003.

13. Hanson C, Strawser D. Family presence during cardiopulmonary resuscitation: Foote Hospital emergency department's nine-year perspective. 7 Emerg Nurs 1992;18:104-6.

14. Robinson SM, Mackenzie-Ross S, Campbell Hewson GL, et al. Psychological effect of witnessed resuscitation on bereaved relatives. Lancet 1998;352:614-7, doi:10.1016/ S0140-6736(97)12179-1.

15. Morrison LJ, Kierzek G, Diekema DS, et al. Part 3: ethics: 2010 American Heart Association Guidelines for Cardiopulmonary Resuscitation and Emergency Cardiovascular Care. Circulation 2010;122(18 Suppl 3):S665-75, doi:10. 1161/CIRCULATIONAHA.110.970905.

16. Lippert FK, Raffay V, Georgiou M, et al. European Resuscitation Council Guidelines for Resuscitation 2010 Section 10. The ethics of resuscitation and end-of-life decisions. 
Resuscitation 2010;81:1445-51, doi:10.1016/j.resuscitation.2010. 08.013.

17. Fulbrook P, Latour J, Albarran J, et al. The presence of family members during cardiopulmonary resuscitation: European Federation of Critical Care Nursing Associations, European Society of Paediatric and Neonatal Intensive Care and European Society of Cardiology Council on Cardiovascular Nursing and Allied Professions joint position statement. Nurs Crit Care 2007;12:250-2, doi:10.1111/j.1478-5153.2007.00240.x.

18. Jabre P, Belpomme V, Azoulay E, et al. Family presence during cardiopulmonary resuscitation. N Engl 7 Med 2013; 368:1008-18, doi:10.1056/NEJMoa1203366.

19. Horowitz M, Wilner N, Alvarez W. Impact of Event Scale: a measure of subjective stress. Psychosom Med 1979;41:209-18.

20. Zigmond AS, Snaith RP. The hospital anxiety and depression scale. Acta Psychiatr Scand 1983;67:361-70, doi:10.1111/ j.1600-0447.1983.tb09716.x.

21. Morton JH. Fathers in the delivery room-an opposition standpoint. Hosp Top 1966;44:103-4.

22. Goetsch C. Fathers in the delivery-"helpful and supportive." Hosp Top 1966;44:104-5.

23. Bradley RA. Fathers' presence in delivery rooms. Psychosomatics 1962;3:474-9, doi:10.1016/S0033-3182(62) 72633-2.
24. Fallis WM, McClement S, Pereira A. Family presence during resuscitation: a survey of Canadian critical care nurses' practices and perceptions. Dynamics 2008;19:22-8.

25. Porter J, Cooper SJ, Sellick K. Attitudes, implementation and practice of family presence during resuscitation (FPDR): a quantitative literature review. Int Emerg Nurs 2013;21:2634, doi:10.1016/j.ienj.2012.04.002.

26. Halm MA. Family presence during resuscitation: a critical review of the literature. Am 7 Crit Care 2005;14:494-511.

27. Critchell CD, Marik PE. Should family members be present during cardiopulmonary resuscitation? A review of the literature. Am 7 Hosp Palliat Care 2007;24:311-7, doi:10. 1177/1049909107304554.

28. Walker W. Accident and emergency staff opinion on the effects of family presence during adult resuscitation: critical literature review. 7 Adv Nurs 2008;61:348-62, doi:10.1111/ j.1365-2648.2007.04535.x

29. Rodwin MA. French medical malpractice law and policy through American eyes: what it reflects about public and private aspects of American law. Legal Studies Research Paper Series 2011;4:100-31.

30. Maciejewski PK, Zhang B, Block SD, Prigerson HG. An empirical examination of the stage theory of grief. $7 A M A$ 2007;297:716-23, doi:10.1001/jama.297.7.716.

31. American Psychiatric Association. Diagnostic and statistical manual of mental disorders. 5th ed. Arlington (VA): American Psychiatric Publishing; 2013. 